\title{
1 Microbes are potential key players in the evolution of life histories and aging in
}

\section{Caenorhabditis elegans}

3

4 Josiane Santos ${ }^{1}$, Margarida Matos ${ }^{1}$, Thomas Flatt ${ }^{2}$, Ivo M Chelo ${ }^{1}$

5

$6{ }^{1}$ cE3c - Centre for Ecology, Evolution and Environmental Changes, Faculdade de Ciências,

7 Universidade de Lisboa, Lisboa, Portugal

8

$9 \quad 2$ Department of Biology, University of Fribourg, Fribourg, Switzerland

10

11 Correspondence: Ivo M Chelo (immchelo@fc.ul.pt)

12

13

14

15

16

17

18

19

20

21 


\section{Abstract}

24 Microbes can have profound effects on host fitness and health and the appearance of late-

25 onset diseases. Host-microbe interactions thus represent a major environmental context for

26 healthy aging of the host and might also mediate trade-offs between life-history traits in the

27 evolution of host senescence. Here we have used the nematode Caenorhadbitis elegans to

28 examine whether host-microbe interactions might modulate the evolution of host life-history

29 and aging. We first characterized the effects of two non-pathogenic Escherichia coli strains, a

30 pathogenic E. coli strain and a pathogenic Serratia marcescens strain on the reproductive

31 schedule and survival of an outbred C. elegans population, to be used in an experimental

32 evolution study. Secondly, to investigate the dependency of these effects on host genotype,

33 we assayed population growth rates and survival of five representative C. elegans inbred

34 strains in response to these microbes. Our results show that host-microbe interactions have a

35 substantial, host-genotype-dependent impact on reproductive effort and survival of the

36 nematode host. Although pathogenic bacteria reduced host survival, as expected, they did not

37 necessarily decrease host fertility or population growth rate. Given such microbe-specific

38 genotypic differences in host life history, we predict that the evolution of reproductive

39 schedules and senescence in this system might be critically contingent upon host-microbe

40 interactions, a hypothesis which we will be testing using experimental evolution in future

41 work.

43 Key words: C. elegans; microbes; host-microbe interactions; life-history evolution; aging;

44 trade-offs. 


\section{Introduction}

49 Microbes are thought to have major effects on the evolution and speciation of host

50 populations due to their ubiquitous presence and ability to influence host physiology and

51 health (Bordenstein et al. 2001; Zilber-Rosenberg and Rosenberg 2008; McFall-Ngai et al.

52 2013). While microbes are best known for their pathogenic or mutualistic effects, they can

53 also modulate how hosts perceive and respond to stressful conditions. This has been

54 observed, for example, in contexts as diverse as viral infections (Martinez et al. 2014), the

55 autoimmune response (Langan et al. 2019), drug therapy (Pryor et al. 2019), metabolic

56 dysfunction (Ussar et al. 2016), exposure to high temperatures (Xie et al. 2013; Howells et al.

57 2016), and chemical toxicity (Coryell et al. 2018). Microbes can therefore impact adaptation

58 of host populations to conditions that are apparently unrelated to the host-microbe interaction

59 itself (Martinez et al. 2016; Faria et al. 2016; Bates et al. 2021; Hoang et al. 2021). This

60 ability of microbes to modulate host adaptation to various stressors might thus play a

61 fundamental but still poorly understood role in shaping the evolution of host life history and

62 aging.

63 The progressive loss of physiological function leading to a decline in fecundity and

64 increased mortality, which defines aging, can be explained by the reduced efficacy of

65 selection in purging mutations that have deleterious effects late in life (Fisher 1930; Haldane

66 1941; Medawar 1946, 1952; Williams 1957; Hamilton 1966; Rose 1991; Kirkwood and

67 Austad 2000; Flatt and Schmidt 2009; Flatt and Partridge 2018). A major mechanism

68 underlying the evolution of aging is antagonistic pleiotropy, i.e., the existence of alleles with

69 antagonistic effects upon early and late life-history traits which lead to genetic trade-offs

70 between fitness components (Medawar 1946, 1952; Williams 1957; Stearns 1989; Rose 1991;

71 Flatt and Promislow 2009; Flatt 2020). Under this model, aging evolves because strong

72 selection for beneficial fitness effects early in life outweighs the deleterious effects of these 
73 alleles late in life when selection is weak (e.g., Williams 1957). A large body of work in

74 numerous organisms, for example the nematode worm Caenorhabditis elegans (Anderson et al. 2011), the fruit fly Drosophila melanogaster (reviewed in Flatt 2020), or the fish Poecilia reticulata (Reznick et al. 1990), has revealed antagonistic pleiotropy underlying trade-offs by showing correlated responses to selection in major fitness components such as developmental

78 rate, early and late-fecundity, and lifespan.

79 Even when populations harbor genetic variation sustaining antagonistic pleiotropic effects,

80 they may not always experience phenotypic trade-offs nor correlated responses of life-history

81 traits to selection, as these also depend on environmental factors (Giesel et al. 1982; Stearns

82 1989; Ackermann et al. 2001; Sgró and Hoffmann 2004; Gutteling et al. 2007; Swanson et al.

83 2016). For this reason, microbes are likely to play a relevant but underappreciated role in the

84 evolution of aging, especially given their known effects on life-history traits (Little et al.

85 2002; Deckaester et al. 2003; Brummel et al. 2004; Vale and Little 2012; Leroy et al. 2012;

86 Laughton et al. 2014; Parker et al. 2014; Diaz et al. 2015; Zurowski et al. 2020) and their

87 evolution (Sorci and Colbert 1995; Gibson et al. 2015; Walters et al. 2020). Causal

88 relationships between the composition of the intestinal microbiome and aging observed in

89 humans (Claesson et al. 2011) and other organisms (Clark et al. 2015; Sonowal et al. 2017;

90 Bárcena et al. 2019) are consistent with this notion.

91 Studies with the C. elegans model hold great promise for an improved understanding of

92 the interplay between host-microbe interactions and the evolution of aging. For example, the

93 worm system has been extensively used in the identification of the genetic pathways

94 underpinning aging and longevity (Garsin et al. 2003; Kurz and Tan 2004; Antebi 2007;

95 Evans et al. 2008; Leroy et al. 2012), many of which are shared with humans (Kurz and Tan

96 2004). At the same time, C. elegans has also been a valuable tool for studying host-microbe

97 interactions (Tan et al. 1999; Abbalay et al. 2000; Garsin et al. 2003; Schulenburg et al. 2004; 
98 Coolon et al. 2009; Leroy et al. 2012; Diaz et al. 2015; Dirksen et al. 2016; Schulenburg and

99 Félix 2017) and how such interactions regulate host development, reproduction, metabolism,

100 immunity and lifespan (MacNeil et al. 2013; Pang and Curran 2014; Chan et al. 2019 ).

101 Notably, links between immunity and aging have been established in C. elegans (Kurz and

102 Tan 2004), for example in the context of lifespan expansion obtained with specific bacterial

103 metabolites (Virk et al. 2012; Han et al. 2017) or by transferring worms from their regular

104 food source (Escherichia coli OP50) to other bacteria such as Bacillus subtilis (Aballay et al.

105 2000; Portal-Celhay et al. 2012; Donato et al. 2017). Moreover, long-lived C. elegans

106 mutants have been found to be resistant to pathogenic bacteria such as Enterococcus faecalis

107 and Staphylococcus aureus (Garsin et al. 2003).

108 The extent to which the evolution of life histories and senescence in the nematode host

109 might be contingent upon specific host-microbe interactions remains poorly understood. To

110 begin to address this question, we sought to examine how different pathogenic and non-

111 pathogenic bacteria impact the reproductive schedule and survival of C. elegans. To this end,

112 we first measured survival and fertility throughout the reproductive lifespan of a genetically

113 diverse population of C. elegans in response to two non-pathogenic E. coli strains, a

114 pathogenic E. coli strain and a pathogenic Serratia marcescens strain. Second, we sought to

115 investigate whether microbial effects on host life history might depend on host genotype, i.e.,

116 whether host life history might be affected by interactions between microbe strain, host

117 genotype and environment (i.e., reproductive timing). To do so, we assayed the population

118 growth rates of five isogenic C. elegans strains, derived from different wild isolates, at the

119 beginning of the reproductive period and past the reproductive peak in response to the same

120 four bacterial strains mentioned above. Our results demonstrate that host-microbe interactions

121 can have profound, host-genotype-dependent effects on reproductive effort and survival in $C$.

122 elegans. Based on these results we conjecture that bacterial symbionts can modulate the 
outcome of host life-history evolution in response to selection for different reproductive

124 schedules, a prediction which we aim to test with experimental evolution in future work.

126 Methods

\section{BACTERIAL STRAINS}

128 Bacterial strains used in our experiments included two commonly employed non-pathogenic

129 Escherichia coli strains, OP50 (Brenner 1974) and HT115(DE3) (Timmons et al. 2001), and

130 two pathogenic strains, E. coli IAI1 (Picard et al. 1999; Diard et al. 2007) and Serratia

131 marcescens Db11 (Flyg et al. 1980; Kurz et al. 2003). E. coli HT115(DE3) had been used as

132 food during the establishment of the C. elegans D00 population described below. The strains

133 E. coli HT115(DE3), E. coli OP50, and S. marcescens Db11 were obtained from the

134 Caenorhabditis Genetics Center (CGC), and the E. coli IAI1 strain was kindly provided by

135 Ivan Matic.

\section{NEMATODE POPULATIONS}

137 To assay life-history responses to the above-mentioned microbe strains we used an outbred

138 experimental C. elegans population (D00) and 5 wild isolates (N2, CB4852, CB4855,

139 CB4856, PX174). The D00 population was first described by Theologidis et al. (2014); it is a

140 genetically diverse dioecious population with males and females, established by introgression

141 of the fog-2(q71) mutant allele (Schedl and Kimble 1988) into the genetic background of a

142 previously laboratory-adapted androdioecious population consisting of males and

143 hermaphrodites (Teotónio et al. 2012; Chelo and Teotónio 2013). Throughout laboratory

144 adaptation, worms were provided with E. coli HT115(DE3) as a food source and evolved

145 under discrete (non-overlapping) generations imposed by a 4-day life-cycle, herein referred to

146 as "early reproduction”. The D00 population is characterized by obligate outcrossing; its

147 standing genetic variation results from an initial mixture of 16 isogenic strains, which were 
148 chosen to represent a significant proportion of the known genetic diversity in C. elegans

149 (Rockman and Kruglyak 2009; Teotónio et al. 2012). Here we have analyzed 5 of these 16

150 isolates (N2, CB4852, CB4855, CB4856, PX174).

\section{GROWTH CONDITIONS}

152 Bacteria were grown overnight in NGM-lite solid media at $37^{\circ} \mathrm{C}$ from LB-grown cultures.

153 Nematode maintenance followed previously described protocols (Stiernagle 1999; Chelo

154 2014). On day one, L1 larvae were seeded on NGM-lite supplemented with ampicillin (100

$155 \mathrm{mg} / \mathrm{ml}$ ), carrying a confluent lawn of E. coli HT115(DE3). $10^{3}$ larvae were used per plate,

156 and development proceeded at $20^{\circ} \mathrm{C}$ and $80 \%$ (RH) for 72 hours, until day four of the life-

157 cycle. Plates were washed with M9 buffer and a KOH:sodium hypochlorite solution was

158 added ("bleaching”) to kill adults and larvae but allowing unhatched embryos to survive.

159 Eclosion of first-stage larvae (L1) occurred overnight in $4 \mathrm{ml}$ of M9 buffer with $2.5 \mathrm{mg} / \mathrm{ml}$ of

160 tetracycline under constant shaking.

161 REPRODUCTIVE SCHEDULE AND SURVIVAL OF THE D00 POPULATION

162 Daily offspring number and survival were monitored to study the effects of different bacteria

163 on individuals of the D00 population. Frozen $\left(-80^{\circ} \mathrm{C}\right)$ stock populations were thawed and

164 maintained for two generations prior to the assay. To set up the experiment, $10^{3} \mathrm{~L} 1$

165 individuals were seeded on NGM-lite plates carrying each of the four bacteria and incubated

166 until the beginning of day 3 (48 hours later). From each plate, 30 female larvae were placed

167 (one larva per well) onto 24-well plates with antibiotic-free NGM-lite and matching bacteria,

168 which had been grown from a $5 \mu$ inoculum. Adult males from the same population and

169 conditions, but which had been developing for one extra day, were added to the wells (two

170 males per well). Individuals were transferred to fresh medium every 12 hours until day 6, and

171 every 48 hours after day 6, until all individuals were found dead or considered to be missing.

172 During the first five days, males that had died (or were missing) were replaced to ensure 
mating and fertilization. After removal of adults, plates were kept in the incubator for one day

174 and then transferred to $4^{\circ} \mathrm{C}$ for a maximum of two days before counting L2-L3 larvae under

175 the stereoscope with 10x-30x magnification. These data were used to determine total fertility

176 (lifetime reproductive success, LRS), variation in fertility through time and the age at first

177 reproduction (AFR). Survival was scored based on daily observations during the entire period

178 of the experiment. Monitoring of missing or dead females occurred at the time of transfer,

179 and individuals were considered dead in the absence of movement or response when being

180 gently touched with a platinum wire.

\section{GROWTH RATE OF THE D00 POPULATION AND OF INDIVIDUAL GENOTYPES}

182 Population growth rate in response to each of the four bacterial strains was measured at two

183 different times: at 72 hours after L1 seed (transition from day 3 to day 4), i.e., within hours of

184 reaching sexual maturity (“early reproduction”; Anderson et al. 2011) and at 114 hours post-

185 seed (day 5; referred to as “delayed reproduction”). Frozen populations were thawed and

186 maintained for two generations under standard maintenance conditions, plus one generation

187 in presence of each bacterial strain for acclimatization. In the fourth generation, L1 larvae

188 were seeded on NGM-lite plates (103/plate) with a lawn of each bacterial strain and allowed

189 to develop for 72 or 114 hours. Following our standard maintenance protocol, cultures were

190 bleached and the number of the live L1s was estimated the following day. Each estimate was

191 obtained by pooling individuals from three plates. The D00 population and each of the five

192 isolates (N2, CB4852, CB4855, CB4856, PX174) were assayed in independent experimental

193 blocks. In the assays with the isolates, each block included the N2 strain feeding on E. coli

194 HT115(DE3) as a common reference, the four different bacteria and the two time points. For

195 each bacterial strain and each time point, we used five technical replicates for D00 and N2

196 and four technical replicates for each of the other four isolates. 


\section{SURVIVAL OF INDIVIDUAL GENOTYPES}

199 The effect of the four bacterial strains on survival was assayed for each of the five C. elegans

200 isolates (CB4852, CB4855, CB4856, PX174). After thaw and growth for two generations

201 under standard maintenance conditions, L1 larvae were seeded on NGM-lite media (10 ${ }^{3}$

202 individuals/plate) with a lawn of each of the four bacteria. 48 hours later (day 3), L4

203 hermaphrodites were placed on 24-well NGM-lite plates (five individuals per well), with the

204 corresponding bacteria, as described above for the survival assay of the D00 population. Each

205 of the four non-N2 isolates was assayed in a different experimental block, which also

206 included included N2 as a common reference. Four plates were used per block, and every

207 plate included all four bacterial strains. Both the N2 and one of the non-N2 isolates were used

208 in every plate, with N2 individuals occupying one fourth of the total number of wells. This

209 experimental design enabled the estimation of plate effects within a block. In total, 480

210 individuals were assayed in each block, with 120 being N2 individuals and 360 individuals

211 from one of the other isogenic strains.

\section{DATA ANALYSIS}

213 Statistical analyses were performed in $R$ ( $R$ Core Team 2019). Supplementary files with

214 analyses and $R$ code can be found at FigShare (see 10.6084/m9.figshare.15022566 for

215 Supplementary Figures; and 10.6084/m9.figshare.15022599 for Supplementary Data and

216 analysis scripts).

217 For fertility data, observations of $12 \mathrm{~h}$ intervals were collapsed into daily measures until

218 day 6 and into a single bin beyond that time. Thus, fertility reported for day 3 refers to

219 embryos laid between $48 \mathrm{~h}$ and $72 \mathrm{~h}$ post-L1 seed, between $72 \mathrm{~h}$ to $96 \mathrm{~h}$ for day 4, between

$22096 \mathrm{~h}$ to $120 \mathrm{~h}$ for day 5, between $120 \mathrm{~h}$ and $144 \mathrm{~h}$ for day 6, and $144 \mathrm{~h}$ onwards to "day 7".

221 Model fitting and model comparisons were performed with generalized linear models with

222 appropriate error distributions (see below), and analysis of deviance was used to test for 
significance. Parameter estimates were retrieved and tested with emmeans and pairs function

224 (Lenth 2018). For pairwise comparisons, we used Tukey’s post-hoc tests and report adjusted

225 -values. The reproductive schedule of the D00 population was modeled with a negative

226 binomial distribution using the $R$ function glm.nb in the MASS package. The following

227 model was used: Fertility $\sim$ Bacteria $*$ Time, where Fertility refers to the number of larvae

228 observed per individual worm during a $24 \mathrm{~h}$ period, Bacteria represents the four bacterial

229 strains tested, and Time is a categorical variable with 5 levels representing the day since the

230 experimental set-up. Post-hoc comparisons were performed between fertility means within

231 each day. Total fertility was modeled with a Poisson distribution using the glm function, as

232 follows: LRS Bacteria, family = "poisson"(link="log”), where LRS is the total number of

233 observed larvae. A Gaussian fit was used to analyze AFR with the following code: AFR

234 Bacteria, family = "gaussian", where AFR (age at first reproduction) refers to the time

235 between L1 seed and the time at which offspring was first observed.

236 Cox regression (proportional hazards analysis; Cox 1972) was used to test for differences

237 in survivorship, with E. coli HT115(DE3) defining the baseline risk. The following model

238 was implemented with the functions Surv and coxph in the survival package in $R$ (Therneau

239 2015): Surv(S.time,S.event) Bacteria, with S.time being the time at which an individual was

240 found dead or missing (S.event), assuming right-censored data. Kaplan-Meier estimation

241 (Kaplan and Meier 1958) was used to estimate survival curves and mean lifespan.

242 Analysis of population growth rate was carried out using the natural logarithm (ln) of the

243 observed rates. Whenever L1 larvae could not be detected, which would lead to growth rate

244 estimates of zero (two samples; see Supplementary Table 2), values were replaced assuming

245 that one L1 had been observed. To standardize the different blocks with C. elegans isolates,

246 the growth rates of $C$. elegans N2 with E. coli HT115(DE3) were first estimated in each

247 block and at each time point with a random-effects model using a block-specific baseline. 
248 The following model was implemented in $R$ : $\log ($ GrowthRate $) \sim$ Time * Bacteria *

249 Celegans, offset=Block_offset, where GrowthRate is the observed L1 growth rate in

250 consecutive generations, Time is the number of hours since L1 seed, Bacteria represents the

251 bacterial strains, Celegans represents the 5 different isolates, and Block_offset is the value of

252 the block effects obtained with N2 and E. coli HT115(DE3).

253 Survival analysis of the C. elegans isolates was performed with Cox regression, using

254 mixed-effect models with the coxme function in $R$ (Thernau 2020) in order to include plate

255 effects. The following model was used: Surv (S.time,S.event) $~$ Bacteria * Celegans $+(1 \mid$

256 Plate) + Block_offset (see above). Mean lifespan values based on Kaplan-Meier estimation

257 were corrected by the values obtained for each block with N2 (see Supplementary Fig. 3).

DIFFERENT BACTERIA HAVE SPECIFIC EFFECTS ON THE LIFE-HISTORY

\section{SCHEDULE OF A GENETICALLY DIVERSE C. ELEGANS POPULATION}

262 Reproduction and survival of the C. elegans D00 population were affected by the different

263 bacteria in unique ways (Fig. 1). The presence of different bacteria had a significant effect on

264 C. elegans survival ( $p$-value $<0.0001$, see Fig. 1A and Supplementary Fig. 1), with higher

265 mortality risks observed in the presence of the pathogenic strains E. coli IAI1 ( $p=0.0001$ for

266 IAI1 vs. HT115; $p<0.0001$ for IAI1 vs. OP50) and S. marcescens Db11 ( $p=0.02$ for Db11

267 vs. HT115; $p=0.005$ for Db11 vs. OP50). Interestingly, no consistently detrimental (i.e.,

268 pathogenic) effects were observed for fertility, even though fertility did vary with the

269 different bacteria (Fig. 1B-C). Significant differences among bacterial strains were found for

270 lifetime fertility ( $p<0.0001$, Fig. 1B), with the highest brood size being observed with $E$. coli

271 HT115(DE3) (371 \pm 4, mean \pm standard error), followed by E. coli OP50 (185 \pm 2$)$, E. coli 
272 IA1 (177 \pm 2$)$ and $S$. marcescens Db11 which resulted in a markedly reduced lifetime fertility

273 (61 \pm 1). These differences were also reflected in the reproductive schedule (Fig. 1C), as

274 revealed by a significant time by bacteria interaction (likelihood ratio test, $\mathrm{LRT}=42.5$, $\mathrm{df}=$

$27512, p<0.001)$. Although fertility was always maximized at day 4, the relative contribution of

276 offspring produced before and after this peak day was dependent on the bacterial strains. For

277 instance, with E. coli HT115(DE3) the higher mean estimates of fertility observed throughout

278 the entire reproductive lifespan of the host only become statistically significant after day 5

279 (pairwise comparisons, adjusted $p$-values $<0.05$ ). In contrast, the initially diminished fertility

280 of S. marcescens Db11 was no longer different from the majority of values observed with the

281 three E. coli strains from day 4 onwards (Fig. 1C). Interestingly, comparing the start of

282 offspring production of $S$. marcescens Db11 with the ones from all E. coli indicates a delay in

283 reproduction, suggesting a possible interference with C. elegans development (Fig. 1D).

\section{BACTERIA-HOST GENOTYPE INTERACTIONS AFFECT C. ELEGANS}

\section{POPULATION DYNAMICS}

286 The differential effects of the bacterial strains on the fertility dynamics of the host shown in

287 Fig. 1C above suggest that the outcome of selection for early reproduction (reproduction at

$28872 \mathrm{~h}$ ) versus delayed reproduction (114 h), and hence the evolution of lifespan, might depend

289 critically on microbe-host genotype interactions. Indeed, we found that the population growth

290 rate (i.e., a fitness proxy) of the D00 population at those two ages was dependent on the

291 bacterial strains (Fig. 2A): this was revealed by a significant time by bacteria interaction

$292(\mathrm{LRT}=2.58, \mathrm{df}=3, p<0.001)$, with different slopes for $E$. coli IAI1 (CI $=-0.028$ to -0.014

293 per hour), E. coli HT115(DE3) (CI = -0.011 to 0.002 per hour), E. coli OP50 (CI = -0.009 to

2940.004 per hour), and S. marcescens Db11 (CI $=0.006$ to 0.0120 per hour). The main effects

295 of time $(\mathrm{LRT}=0.25, \mathrm{df}=1, p=0.03)$ and bacterial strain $(\mathrm{LRT}=1.89, \mathrm{df}=3, p<0.0001)$

296 were also significant. 
Importantly, the time-bacteria interaction effects on growth rates also varied among the

298 five C. elegans genotypes, as revealed by the pervasive crossing of lines in Fig. 2B and

299 confirmed by a significant three-way interaction term $(\mathrm{LRT}=17.71 \mathrm{df}=12, p<0.0001)$

300 (main effects and all two-way interactions were also significant, not shown). Individual plots

301 by bacterial strain (Supplementary Fig. 2) indicate that these effects were comparable to those 302 obtained for the D00 population. This can be seen, for example, with E. coli IAI1 which

303 imposed the largest average decrease in growth rate with time $(C I=-0.097$ to -0.084 per

304 hour), or with $S$. marcescens Db11 which caused a shallower slope (CI $=-0.015$ to -0.003 per

305 hour). In this latter case, it is noteworthy that the usual reduction of growth rate with time

306 was reversed for the CB4855 genotype in presence of S. marcescens Db11 (Supplementary

307 Fig. 2D).

308 BACTERIA-HOST GENOTYPE INTERACTIONS MODULATE C. ELEGANS

309 LIFESPAN

310 The different bacteria also affected the adult survival of the C. elegans isolates (Fig. 2C and

311 Supplementary Fig. 3, $x=629.6, \mathrm{df}=3, p<0.001$ ), with a significant bacteria-host genotype 312 interaction on lifespan $(x=72.9, \mathrm{df}=12, p<0.0001)$. Interestingly, for one of these isolates

313 we also observed a departure from the overall deleterious effect of the pathogenic bacteria on

314 lifespan: for the PX174 genotype, lifespan in presence of $S$. marcescens (8.7 \pm 0.2 days) was 315 clearly not lower than in presence of E. coli HT115(DE3) (8.1 \pm 0.4 days).

\section{Discussion}

318 Aging represents one of the most compelling examples in evolutionary biology of how trait

319 optimization can have detrimental side effects leading to physiological dysfunction. Here,

320 using the nematode model C. elegans, we have confirmed that microorganisms can shape the 
321 host environment in which life-history traits are expressed by showing that distinct bacterial

322 strains have specific effects on host survival, lifetime fertility and reproductive schedule (Fig.

323 1). Importantly, we also observed that different strains affect the nematode's reproductive

324 dynamics and survival in a host-genotype-specific manner, suggesting that such bacteria-host

325 interactions might affect the evolution of aging in the host.

326 Given the diverse type of interactions that bacteria can establish with C. elegans (Diard et

327 al. 2007; Abalay et al. 2009; Coolon et al. 2009; Baeriswyl et al. 2010; Diaz et al. 2015;

328 Dirksen et al. 2016; Stuhr and Curran 2020), the observed differences in life-history

329 responses to specific bacterial strains (Fig. 1) were not entirely surprising. Nevertheless, the

330 observed bacterial effects did not follow simple expectations based on our knowledge of

331 strain pathogenicity. For instance, while the pathogenic bacteria E. coli IAI1 and $S$.

332 marcescens Db11 had clear detrimental effects on host survival, they did not affect fertility;

333 similarly, age at first reproduction was unaffected in presence of E. coli IAI1. Another

334 illustration of this are E. coli IAI1 and E. coli OP50, which imposed the highest and lowest

335 death rates (Fig. 1A), respectively, but which had similar effects on lifetime fertility (Fig.

336 1B), in agreement with previous findings (Baeriswyl et al. 2010). Moreover, the recent

337 evolutionary history of the D00 population might also explain some of the observed patterns:

338 E. coli HT115(DE3), the bacterium used as the food source during the previous 140

339 generations of laboratory adaptation (Teotónio et al. 2012; Chelo and Teotónio 2013;

340 Theologidis et al. 2014), led to the highest lifetime fertility observed.

341 The apparent decoupling of the effects of pathogenic bacteria on C. elegans reproduction

342 and survival (also see Diaz et al. 2015) are consistent with the existence of a degree of

343 specificity in how bacteria interact with their nematode host's physiology, such that

344 development, metabolism or immunity can be affected independently (Coolon et al. 2009;

345 MacNeil et al. 2013, Maynard and Weinkove 2020). This may also explain why C. elegans 
survival can differ considerably even between bacteria that are generally regarded as benign (Brooks et al. 2009; Baeriwisyl et al. 2010; Reinke et al. 2010; Pang and Curran 2014).

Our observation that the time-dependence of host population growth and survival differs among different host isolates in a bacterial-dependent way (Fig. 2) strongly suggests that the evolution of life-history traits might be subject to microbial modulation. In this context, bacteria can be regarded as alternative environments where the fitnesses (here given by population growth rate) of the different genotypes are ranked in an environment-specific manner.

Our results are consistent with several lines of independent evidence showing that in $C$. elegans and/or in other organisms: (i) microbes can influence the expression of life-history traits (Coolon et al. 2009; Storelli et al. 2011; Diaz et al. 2015); (ii) genotype-by-age effects are common (Leips et al. 2006; Viñuella et al. 2010); and (iii) genetic correlations between life-history traits can be subject to modulation by environmental (external) factors (Giesel et al. 1982; Stearns 1989, 1992; Gutteling et al. 2007; Swanson et al. 2016).

A demonstration of a major causal role of microbes as environmental determinants of the evolution of aging could be obtained with an experimental evolution experiment based on selection for delayed reproduction (Figure 3), an approach we are currently taking with $C$. elegans. In such an experiment, different bacterial strains might change the genetic correlations between life-history traits expressed early and late in life. Such an approach might be able to reveal to what extent the expression of genetic life-history trade-offs and the evolution of aging, subject to such trade-offs, are constrained by microbial effects.

\section{AUTHOR CONTRIBUTIONS}

M.M., T.F. and I.M.C. conceptualized and designed the project. J.S and I.M.C. collected and analyzed the data. J.S., M.M., T.F. and I.M.C. interpreted the data and wrote the manuscript. 


\section{ACKNOWLEDGMENTS}

373 We are grateful to Patrícia Beldade and Sara Magalhães for helpful comments on a previous

374 version of the manuscript. Nematode strains were provided by the Caenorhabditis Genetics

375 Center (CGC), funded by the NIH Office of Research Infrastructure Programs (P40

376 OD010440); the E. coli IAI1 strain by Ivan Matic; and the D00 population by Henrique

377 Teotónio. Our research was supported by the FCT (Fundação para a Ciência e Tecnologia;

378 grants IF/00031/2013 and PTDC/BIA-EVL/28757/2017 to I.M.C, and grant

379 SFRH/BPD/123405/2016 to J.S.) and by cE3c unit funding (UIDB/00329/2021). We also

380 acknowledge the Instituto Gulbenkian de Ciência (IGC), where the initial experiments were

381 performed, in particular the support through the ONEIDA project (LISBOA-01-0145-

382 FEDER-016417).

\section{FIGURE LEGENDS}

386 Figure 1. Different bacteria have specific effects on the reproductive output and survival of

387 the genetically diverse C. elegans D00 host population. (A) shows hazard rates imposed by

388 the benign E. coli HT115(DE3) and E. coli OP50 strains or the pathogenic E. coli IAI1 and S.

389 marcescens Db11 bacteria (means plus standard errors SE ). (B) shows lifetime reproductive

390 success; and (C) shows the reproductive schedule. (D) display results for age at first

391 reproduction, given in hours and days after L1 seed (for comparison with other panels in the

392 figure). Shown are means plus SE. Letters above bars indicate group assignment based on

393 post-hoc tests (adjusted $p$-value $<0.05$, see Methods), which in $(\mathbf{C})$ were performed within

394 each time period. 
396 Figure 2. Genotype-by-environment (bacteria) interactions affect C. elegans population

397 growth and survival. In (A), population growth rates of the genetic variable D00 population,

398 measured at the early (72 h) and delayed reproduction period (114 h), reveal bacteria-specific

399 effects on the temporal dynamics of reproductive output . Similarly, in (B), differences

400 between population growth at both times are bacterial dependent (color code as in (A)), but

401 specific for each of the five C. elegans genotypes) (significant three-way interaction, $p$-value

$402<0.001)$. (C) shows that mean lifespan depends on the interaction between C. elegans

403 genotype and bacterial type. Letters above symbols show group assignment from significant

404 post-hoc tests ( $p$-value < 0.05) obtained with data for each bacterium independently. Mean

405 estimates and SE are shown in (A) and (B); predicted values are shown in (C). Note the

406 logarithmic scale of the $y$ axis in (A) and (B).

407

408 Figure 3. Experimental evolution scenarios. Using experimental evolution, different

409 scenarios of life-history evolution may be obtained by comparison with the effects of delayed

410 reproduction in the ancestral population. The figure depicts different hypothetical

411 evolutionary outcomes. The white line shows hypothetical phenotypic values of C. elegans

412 populations feeding on a reference bacterial strain (control); black lines show measurements

413 taken while feeding on another strain (pathogenic or benign). In (A), host-microbial

414 interactions (HM) reveal the interplay between reproductive timing and bacterial type for the

415 genetically diverse ancestral population. These values are displayed as dashed lines in other

416 plots for comparison. Comparing the ancestral population with derived populations

417 maintained under control conditions (B) or selected for delayed fecundity (C) in the presence

418 of different bacteria should allow understanding the role of C. elegans-bacteria interactions

419 in the evolution of life-history and aging phenotypes (C), by first accounting for adaptation to

420 the different bacteria (B vs. C). 


\section{LITERATURE CITED}

423 Aballay, A., P. Yorgey, and F. M. Ausubel. 2000. Salmonella typhimurium proliferates and establishes a persistent infection in the intestine of Caenorhabditis elegans. Curr. Biol. 10:1539-1542.

Anderson, J. L., R. M. Reynolds, L. T. Morran, J. Tolman-Thompson, and P. C. Phillips. 1300-1308.

Antebi, A. 2007. Genetics of aging in Caenorhabditis elegans. PLoS Genet. 3:e129. progeroid mice. Nat. Med. 25:1234-1242. experimental host evolution. Mol. Ecol. 15:3882-3892.

444 Bordenstein, S. R., F. P. O’Hara, and J. H. Werren. 2001. Wolbachia-induced incompatibility precedes other hybrid incompatibilities in Nasonia. Nature 409:707-710. 
446 Brenner, S. 1974. The genetics of Caenorhabditis elegans. Genetics 77:71-94.

447 Brooks, K. K., B. Liang, and J. L. Watts. 2009. The influence of bacterial diet on fat storage in C. elegans. PLoS One 4:e7545.

449 Brummel, T., A. Ching, L. Seroude, A. F. Simon, and S. Benzer. 2004. Drosophila lifespan enhancement by exogenous bacteria. Proc. Natl. Acad. Sci. U. S. A. 101:12974 -

452 Chan, J. P., J. R. Wright, H. T. Wong, A. Ardasheva, J. Brumbaugh, C. McLimans, and R. Lamendella. 2019. Using Bacterial Transcriptomics to Investigate Targets of HostBacterial Interactions in Caenorhabditis elegans. Sci. Rep. 9:1-12.

Chelo, I. M. 2014. Experimental determination of invasive fitness in Caenorhabditis elegans. Nat. Protoc. 9:1392-400.

Chelo, I. M., and H. Teotónio. 2013. The opportunity for balancing selection in experimental populations of Caenorhabditis elegans. Evolution 67:142-56.

Claesson, M. J., S. Cusack, O. O’Sullivan, R. Greene-Diniz, H. De Weerd, E. Flannery, J. R.

465 Clark, R. I., A. Salazar, R. Yamada, S. Fitz-Gibbon, M. Morselli, J. Alcaraz, A. Rana, M.

466 Rera, M. Pellegrini, W. W. Ja, and D. W. Walker. 2015. Distinct shifts in microbiota composition during Drosophila aging impair intestinal function and drive mortality. Cell Rep. 12:1656-1667.

469 Coolon, J. D., K. L. Jones, T. C. Todd, B. C. Carr, and M. A. Herman. 2009. Caenorhabditis elegans genomic response to soil bacteria predicts environment-specific genetic effects 
on life history traits. PLoS Genet. 5: e1000503.

472

473

474

475

476

477

478

479

Coryell, M., M. McAlpine, N. V Pinkham, T. R. McDermott, and S. T. Walk. 2018. The gut microbiome is required for full protection against acute arsenic toxicity in mouse models. Nat. Commun. 9:5424.

Cox, D. R.1972. Regression models and life tables. J R Stat Soc B 34:187-220.

Decaestecker, E., A. Vergote, D. Ebert, and L. De Meester. 2003. Evidence for strong host clone-parasite species interactions in the Daphnia microparasite system. Evolution 57:784-792.

Diard, M., S. Baeriswyl,O. Clermont,S. Gouriou,B. Picard,F. Taddei,E. Denamur, and I. Matic 2007. Caenorhabditis elegans as a simple model to study phenotypic and genetic virulence determinants of extraintestinal pathogenic Escherichia coli. Microbes Infect. 9:214-223.

Diaz, A. S., E. Q. Mooring, E. G. Rens, and O. Restif. 2015. Association with pathogenic bacteria affects life-history traits and population growth in Caenorhabditis elegans. Ecol. Evol. 5:1653-1663.

Dirksen P, S. A. Marsh, I. Braker, N. Heitland, S. Wagner, R. Nakad, S. Mader, C. Petersen., V. Kowallik, P. Rosenstiel, M.-A. Félix, and H. Schulenburg. 2016. The native microbiome of the nematode Caenorhabditis elegans: gateway to a new hostmicrobiome model. BMC Biol. 14:38.

Donato, V., F. R. Ayala, S. Cogliati, C. Bauman, J. G. Costa, C. Leñini, and R. Grau. 2017. Bacillus subtilis biofilm extends Caenorhabditis elegans longevity through downregulation of the insulin-like signalling pathway. Nat. Commun. 8:14332.

Evans, E. A., W. C. Chen, and M. W. Tan. 2008. The DAF-2 insulin-like signaling pathway independently regulates aging and immunity in C. elegans. Aging Cell 7: 879-893.

Faria, V. G., N. E. Martins, S. Magalhães, T. F. Paulo, V. Nolte, C. Schlötterer, É. Sucena, 
and L. Teixeira. 2016. Drosophila adaptation to viral infection through defensive symbiont evolution. PLOS Genet. 12: e1006297.

498 Fisher, R. A. 1930. The genetical theory of natural selection. Oxford at the Clarendon Press, Oxford.

500 Flatt, T. 2020. Life-history evolution and the genetics of fitness components in Drosophila melanogaster. Genetics 214: 3-48.

502 Flatt, T., and L. Partridge. 2018. Horizons in the evolution of aging. BMC Biol. 16: 93.

503 Flatt, T., and D. E. L. Promislow. 2007. Still pondering an age-old question. Science. 318: 1255-1256.

505 Flatt, T., and P. Schmidt. 2009. Integrating evolutionary and molecular genetics of aging. Biochimica et Biophysica Acta 1790:951-962.

507 Flyg, C., K. Kenne, and H. G. Boman. 1980. Insect pathogenic properties of Serratia marcescens: phage-resistant mutants with a decreased resistance to Cecropia immunity and a decreased virulence to Drosophila. J. Gen. Microbiology 120:173-181.

510 Garsin, D. A., J. M. Villanueva, J. Begun, D. H. Kim, C. D. Sifri, S. B. Calderwood, G.

511 Ruvkun, and F. M. Ausubel. 2003. Long-lived C. elegans daf-2 mutants are resistant to bacterial pathogens. Science 300:1921.

513 Gibson, A. K., K. S. Stoy, I. A. Gelarden, M. J. Penley, C. M. Lively, and L. T. Morran. 2015. The evolution of reduced antagonism — A role for host-parasite coevolution.

516 Giesel, J.T., P.A. Murphy, and M.N. Manlove. 1982. The influence of temperature on genetic interrelationships of life history traits in a population of Drosophila

518 melanogaster: what tangled data sets we weave. Am. Nat. 119:464-479.

519 Gutteling, E. W., A. Doroszuk, J. A. G. Riksen, Z. Prokop, J. Reszka, and J. E. Kammenga. 2007. Environmental influence on the genetic correlations between life-history traits in 

unexpected symbiont partners enable reef-building corals to tolerate extreme temperatures. Glob. Chang. Biol. 22:2702-2714.

Kaplan, E. L. and P. Meier. 1958. Nonparametric estimation from incomplete observations. J.

535 Kirkwood, T. B. L., and S. N. Austad. 2000. Why do we age? Nature 408:233-238.

536 Kurz, C. L., S. Chauvet, E. Andrès, M. Aurouze, I. Vallet, G. P. F. Michel, M. Uh, J. Celli, A.

540 Kurz, C. L., and M. Tan. 2004. Regulation of aging and innate immunity in C. elegans. Aging Cell 3:185-193.

542 Langan, D., E. Y. Kim, and K. D. Moudgil. 2019. Modulation of autoimmune arthritis by environmental 'hygiene’ and commensal microbiota. Cell. Immunol. 339: 59-67.

544 Laughton, A. M., M. H. Fan, and N. M. Gerardo. 2014. The combined effects of bacterial symbionts and aging on life history traits in the pea aphid, Acyrthosiphon pisum. Appl. 
Environ. Microbiol. 80:470-477.

547 Leips, J., P. Gilligan, and T. F. C.Mackay. 2006. Quantitative trait loci with age-specific 548 effects on fecundity in Drosophila melanogaster. Genetics 172: 1595-1605.

549 Lenth, R. 2018. emmeans: Estimated marginal means, aka least-squares means. R package version 1.3.1.

551 Leroy, M., T. Mosser, X. Manière, D. F. Alvarez, and I. Matic. 2012. Pathogen-induced Caenorhabditis elegans developmental plasticity has a hormetic effect on the resistance to biotic and abiotic stresses. BMC Evol. Biol. 12:187.

554 Little, T. J., H.-J. Carius, O. Sakwinska, and D. Ebert. 2002. Competitiveness and life-history characteristics of Daphnia with respect to susceptibility to a bacterial pathogen. J. Evol. Biol. 15:796-802.

MacNeil, L. T., E.Watson, H. E. Arda, L. J. Zhu, and A. J. M. Walhout. 2013. Dietinduced developmental acceleration independent of TOR and insulin in C. elegans. Cell 153: $240-252$.

Martinez, J., B. Longdon, S. Bauer, Y.-S. Chan, W. J. Miller, K. Bourtzis, L. Teixeira, and F. M. Jiggins. 2014. Symbionts commonly provide broad spectrum resistance to viruses in insects: a comparative analysis of Wolbachia strains. PLOS Pathog. 10: e1004369. Addicted? Reduced host resistance in populations with defensive symbionts. Proc. R. Soc. B Biol. Sci. 283:20160778. 
Medawar, P. B. 1946. Old age and natural death. Mod. Quart. 2:30-49.

Medawar, P. B. 1952. An Unsolved Problem of Biology. H.K. Lewis, London.

576 Pang, S. and S. P. Curran. 2014. Adaptive capacity to bacterial diet modulates aging in $C$.

Parker, B. J., J. R. Garcia, and N. M. Gerardo. 2014. Genetic variation in resistance and

Picard, B., J. S. Garcia, S. Gouriou, P. Duriez, N. Brahimi, E. Bingen, J. Elion, and E.

$$
\text { extraintestinal infection. Infect. Immun. 67: 546-553. }
$$

Portal-Celhay, C., E. R. Bradley, and M. J. Blaser. 2012. Control of intestinal bacterial

Pryor, R., P. Norvaisas, G. Marinos, L. Best, L. B. Thingholm, L. M. Quintaneiro, W. De Haes, D. Esser, S. Waschina, C. Lujan, R. L. Smith, T. A. Scott, D. Martinez-Martinez, microbe-drug-nutrient screen identifies bacterial effectors of metformin therapy. Cell 178: 1299-1312.e29.

R Core Team. 2019. A Language and environment for statistical computing. R Foundation for Statistical Computing 2. Avalailable at https://www.R--project.org.

Reinke, S. N.,X. Hu, B. D.Sykes, and B. D. Lemire. 2010. Caenorhabditis elegans diet significantly affects metabolic profile, mitochondrial DNA levels, lifespan and brood 
size. Mol. Genet. Metab. 100: 274-282.

597 Reznick, D. A., H. Bryga, and J. A. Endler. 1990. Experimentally induced life-history evolution in a natural population. Nature 346:357-359.

599 Rockman, M., a>nd L. Kruglyak. 2009. Recombinational landscape and population genomics of Caenorhabditis elegans. PLoS Genet 5:e1000419.

601 Rose, M. R. 1991. Evolutionary biology of aging. New York. Oxford University Press.

602 Schedl, T. B. and J. E. Kimble. 1988. fog-2, a germ-line-specific sex determination gene required for hermaphrodite spermatogenesis in C. elegans. Genetics 119: 43-61.

604 605

606

607

608

609

610

Schulenburg, H., C. L. Kurz, and J. J. Ewbank. 2004. Evolution of the innate immune system: the worm perspective. Immunol. Rev. 198:36-58.

Schulenburg, H. and M. A. Félix. 2017. The Natural Biotic Environment of Caenorhabditis elegans. Genetics 206:55- 86.

Sgrò, C. M., and A. A. Hoffmann. 2004. Genetic correlations, tradeoffs and environmental variation. Heredity. 93:241-8.

Sonowal, R., A. Swimm, A. Sahoo, L. Luo, Y. Matsunaga, Z. Wu, J. A. Bhingarde, E. A. Ejzak, A. Ranawade, H. Qadota, D. N. Powell, C. T. Capaldo, J. M. Flacker, R. M. Jones, G. M. Benian, and D. Kalman. 2017. Indoles from commensal bacteria extend healthspan. Proc. Natl. Acad. Sci. 114:7506-7515.

Sorci, G., and J. Clobert. 1995. Effects of maternal parasite load on offspring life-history traits in the common lizard (Lacerta vivipara). J. Evol. Biol. 8:711-723.

Stearns, S. C. 1989. Trade-offs in life-history evolution. Funct. Ecol. 3:259-268.

Stearns, S. C. 1992. The Evolution of Life Histories. Oxford University Press, Oxford.

Stiernagle, T. Maintenance of C. elegans. 2006. WormBook 1-11.

Storelli, G., A Defaye, B. Erkosar, P. Hols, J. Royet, and F. Leulier. 2011. Lactobacillus plantarum promotes Drosophila systemic growth by modulating hormonal signals 
Stuhr, N. L., and S. P. Curran. 2020. Bacterial diets differentially alter lifespan and healthspan trajectories in C. elegans. Commun Biol 3:653.

624 Swanson, E. M., A. Espeset, I. Mikati, I. Bolduc,R. Kulhanek, W. A. White, S. Kenzie, and E. C. Snell-Rood. 2016. Nutrition shapes life-history evolution across species. Proc. R. Soc. B Biol. Sci. 283:20152764. by Pseudomonas aeruginosa used to model mammalian bacterial pathogenesis. Proc. Natl. Acad. Sci. 96:715-720.

630 Teotónio, H., S. Carvalho, D. Manoel, M. Roque, and I. M. Chelo, 2012. Evolution of outcrossing in experimental populations of Caenorhabditis elegans. PLoS One 7:e35811.

Theologidis, I., I. M. Chelo, C. Goy, and H. Teotónio. 2014. Reproductive assurance drives transitions to self-fertilization in experimental Caenorhabditis elegans. BMC Biology, 12:93.

636 Therneau T. 2015. A package for survival analysis in S_ version 2.38.

637 Therneau T. M. 2020. coxme: Mixed effects Cox models. R package version 2.2-16.

638 Timmons, L., D. L. Court, and A. Fire. 2001. Ingestion of bacterially expressed dsRNAs can produce specific and potent genetic interference in Caenorhabditis elegans. Gene 263:103-112. pathogen in Daphnia. J. Evol. Biol. 25:1888-96.

645 Viñuela, A., L. B. Snoek, J. A. G. Riksen and J. E. Kammenga. 2010. Genome-wide gene 
expression regulation as a function of genotype and age in C. elegans. Genome Res. 20:929-937.

648 Virk, B., G. Correia, D. P. Dixon, I. Feyst, J. Jia, N. Oberleitner, Z. Briggs, E. Hodge, R.

649 Edwards, J. Ward, D. Gems, and D. Weinkove. 2012. Excessive folate synthesis limits

650 lifespan in the C. elegans: E. coli aging model. BMC Biol. 10:67-78.

651 Walters, A. W., R. C. Hughes, T. B. Call, C. J. Walker, H. Wilcox, S. C. Petersen, S. M.

652 Rudman, P. D. Newell, A. E. Douglas, P. S. Schmidt, and J. M. Chaston. 2020. The microbiota influences the Drosophila melanogaster life history strategy. Mol. Ecol. 29:639-653.

655 Williams, G. C. 1957. Pleiotropy, natural selection, and the evolution of senescence. Evolution 11:398-411.

657 Xie, B., S. Bishop, D. Stessman, D. Wright, M. H. Spalding, and L. J. Halverson. 2013.

658 Chlamydomonas reinhardtii thermal tolerance enhancement mediated by a mutualistic 659 interaction with vitamin B12-producing bacteria. ISME J. 7:1544-1555.

660 Zilber-Rosenberg, I., and E. Rosenberg. 2008. Role of microorganisms in the evolution of 661 animals and plants: The hologenome theory of evolution. FEMS Microbiol. Rev. 32:723-735.

663 Zurowski, K., A. F. Janmaat, T. Kabaluk, and J. S. Cory. 2020. Modification of reproductive 664 schedule in response to pathogen exposure in a wild insect: Support for the terminal 665 investment hypothesis. J. Evol. Biol. 33:1558-1566. 
(A)
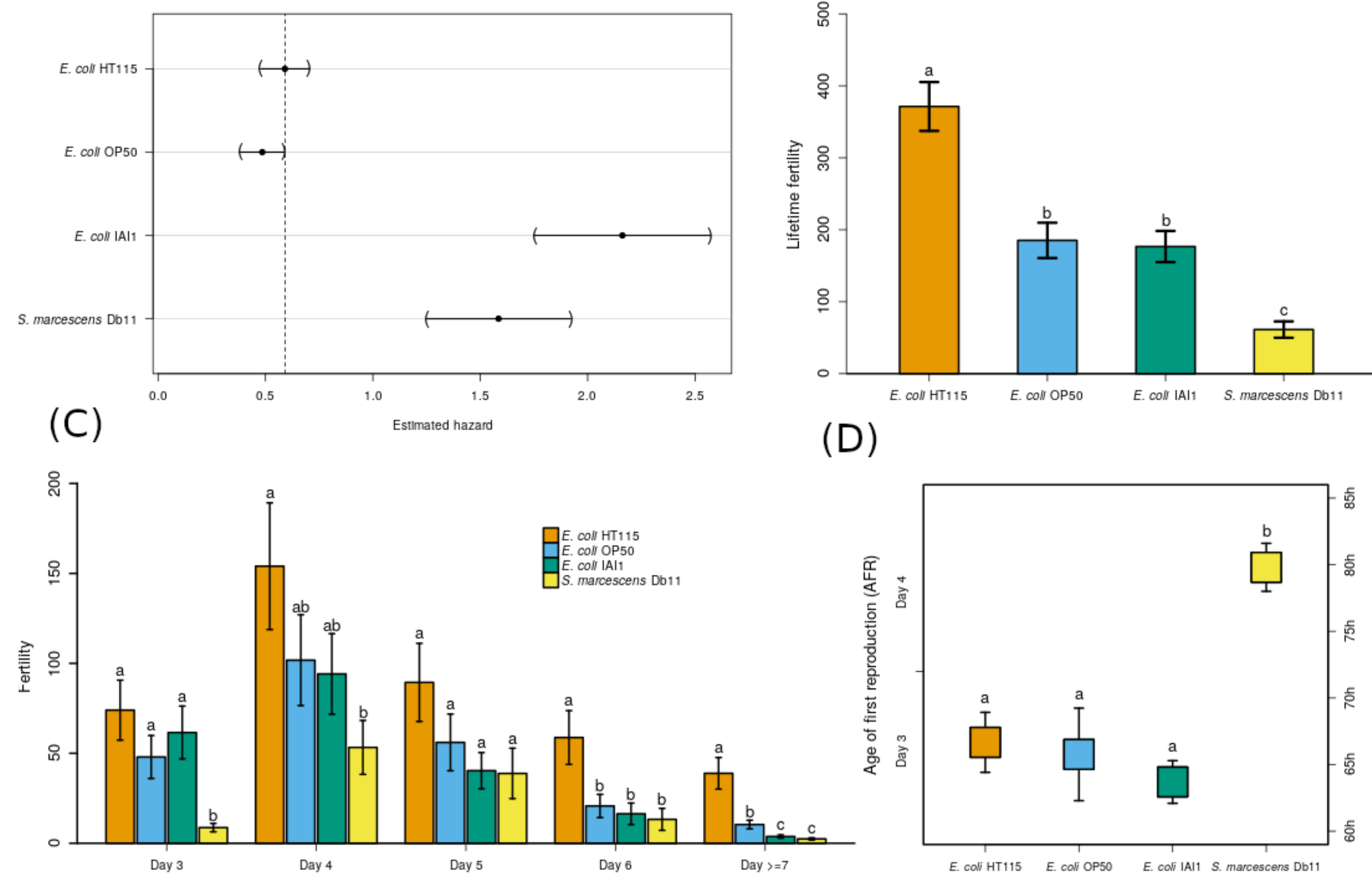

(B)

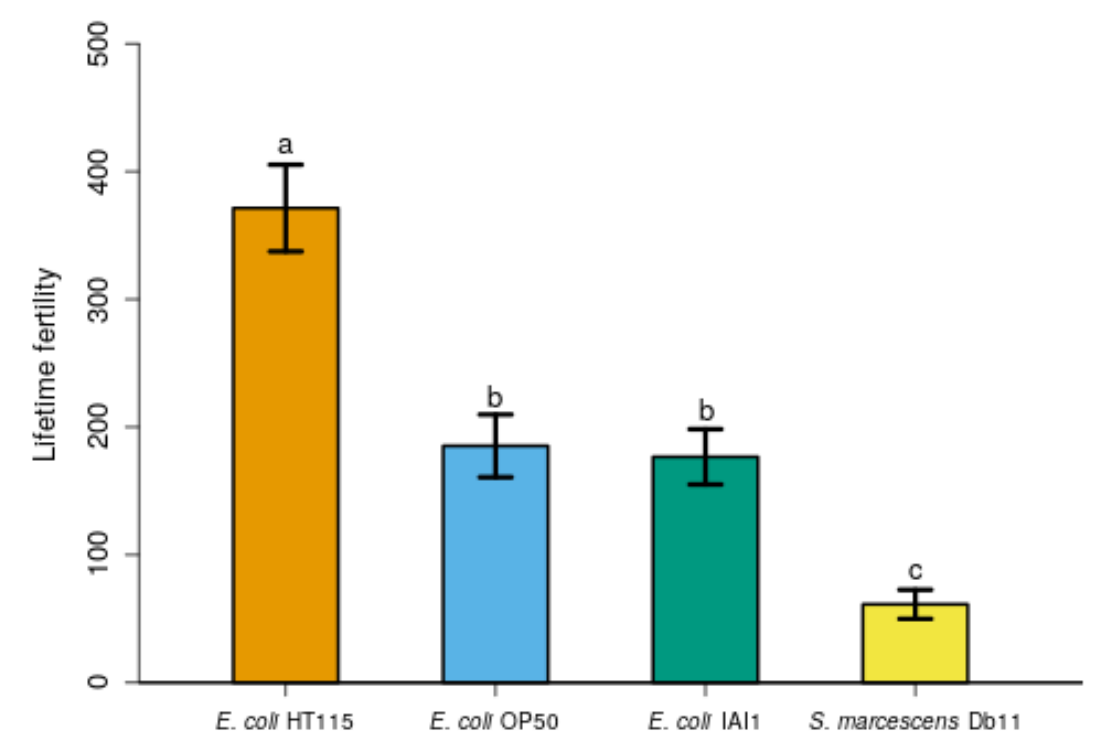

(D) $\begin{array}{llll}\text { E. cali HT115 } & \text { E. coli OP50 } & \text { E. cali IAl1 } & \text { S. marcescens Db11 }\end{array}$

Figure 1.

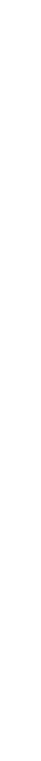



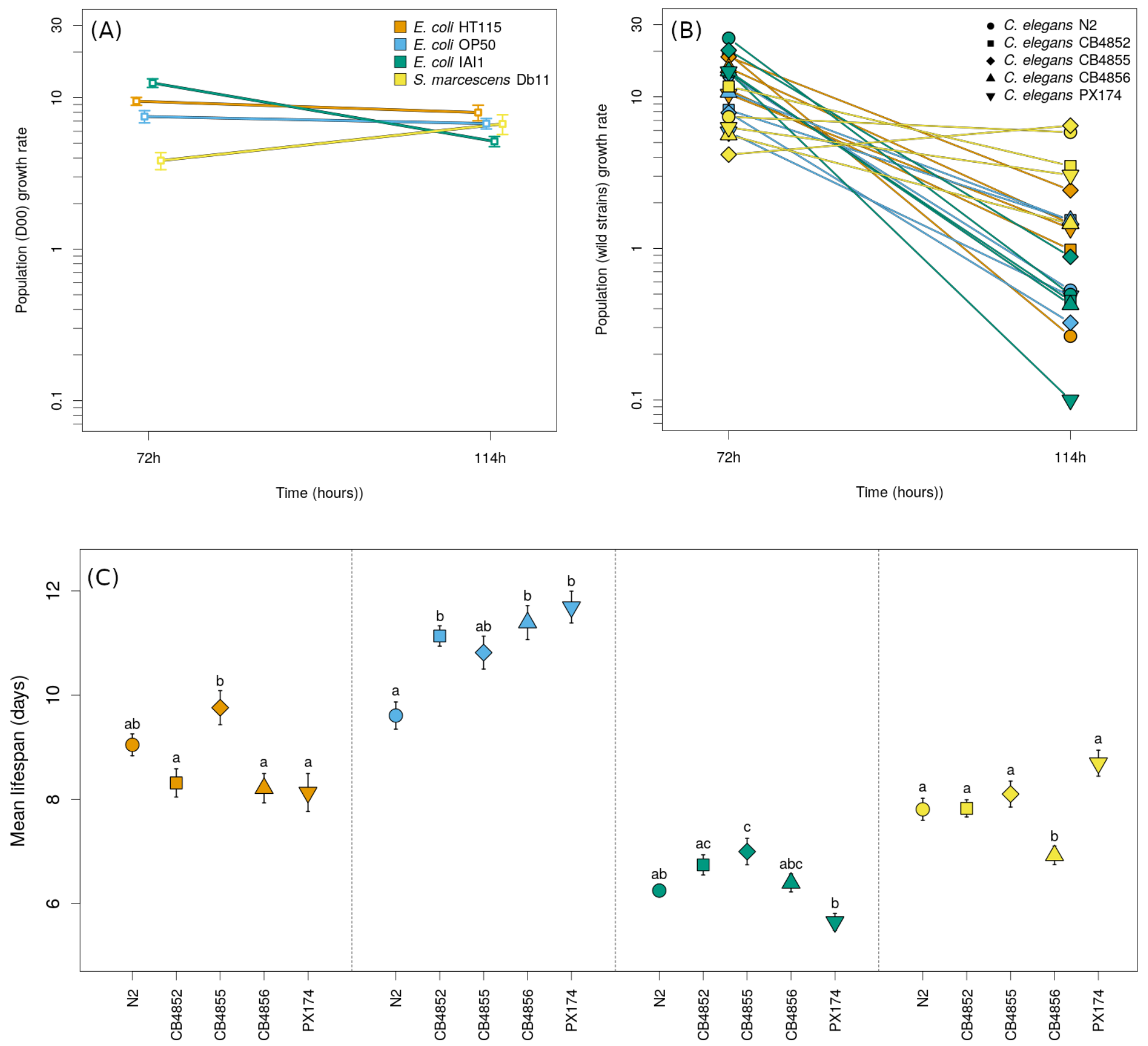

Figure 2. 
(A)

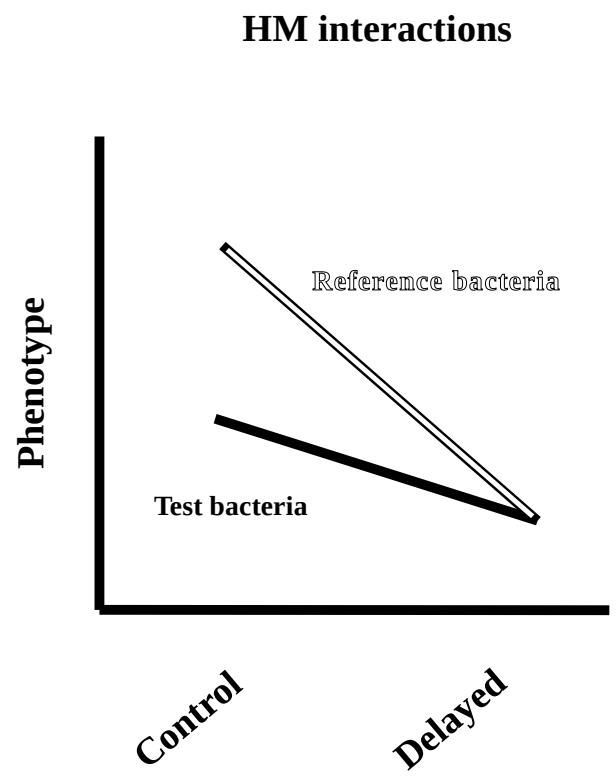

(B)

Host evolution and HM interactions

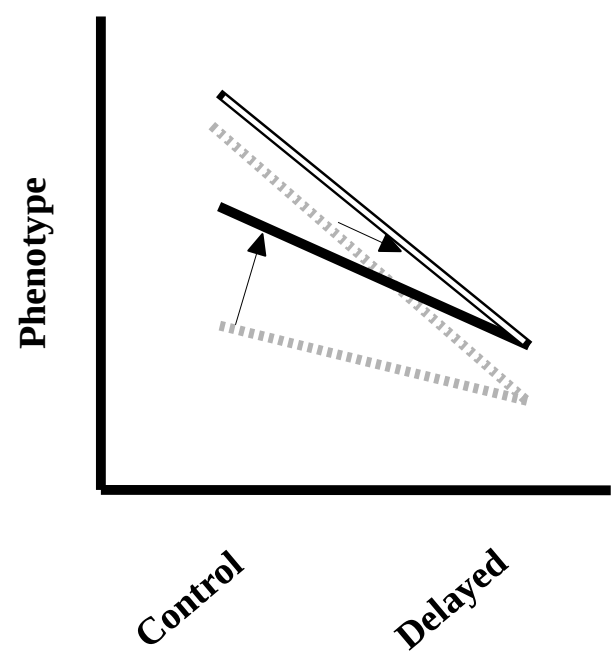

(C)

Host and late-fecundity evolution

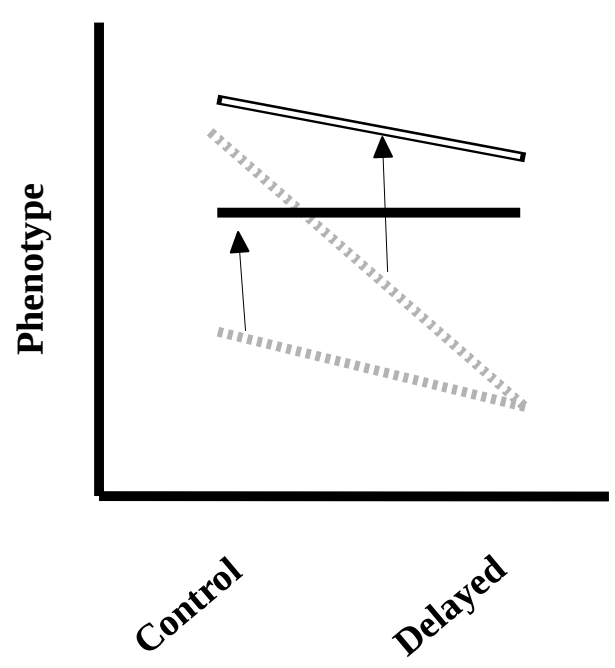

Figure 3. 